\title{
Kernos
}

Revue internationale et pluridisciplinaire de religion grecque antique

$28 \mid 2015$

Varia

\section{Choral Mediations in Greek Tragedy}

\section{Rocco Marseglia}

\section{QpenEdition \\ Journals}

\section{Édition électronique}

URL : http://journals.openedition.org/kernos/2342

DOI : 10.4000/kernos.2342

ISSN : 2034-7871

\section{Éditeur}

Centre international d'étude de la religion grecque antique

\section{Édition imprimée}

Date de publication : 1 octobre 2015

Pagination : 277-280

ISBN : 978-2-87562-055-2

ISSN : 0776-3824

\section{Référence électronique}

Rocco Marseglia, «Choral Mediations in Greek Tragedy », Kernos [En ligne], 28 | 2015, mis en ligne le 01 octobre 2015, consulté le 23 septembre 2020. URL : http://journals.openedition.org/kernos/2342 ; DOI : https://doi.org/10.4000/kernos.2342

Ce document a été généré automatiquement le 23 septembre 2020.

Kernos 


\title{
Choral Mediations in Greek Tragedy
}

\author{
Rocco Marseglia
}

\section{RÉFÉRENCE}

GAGNÉ Renaud, GOVERS HOPMAN Marianne (éd.), Choral Mediations in Greek Tragedy, Cambridge, Cambridge University Press, 2013. 1 vol. $16 \times 23,5 \mathrm{~cm}, \mathrm{x}+429 \mathrm{p}$. ISBN :

978-1-107-03328-3.

1 Dans les dernières décennies, les études sur le chœur dans l'Antiquité grecque ont connu un renouvellement considérable. Dans son étude consacrée aux chœurs de jeunes filles et publiée en $1977^{1}$, Claude Calame montrait que la danse et le chant choral étaient une activité d'ordre rituel et que les chœurs de la Grèce archaïque avaient une fonction sociale et religieuse. Il ouvrait ainsi la voie à un champ d'analyse vaste et fertile et marquait le début d'un nouvel intérêt pour les formes de la poésie chorale. De même, dans le domaine des études théâtrales, les savants se sont davantage intéressés aux chœurs et à leur fonction, dans une approche relativisant la dimension narrative du drame, centrale depuis la lecture aristotélicienne de la tragédie. Ces études ont notamment permis de mettre en lumière la complexité et la variété du chœur tragique, qui, à travers le chant, la danse, la performance masquée, agit sur plusieurs plans en même temps et ne semble pouvoir être réduit à sa dimension fictionnelle, dramatique ou performative. En s'insérant dans ce large débat, ce volume se propose d'offrir des outils méthodologiques pour analyser la capacité qu'a le chœur de combiner plusieurs niveaux de référence et de servir comme un intermédiaire entre eux. C'est là le concept de "choral mediation", que les éditeurs définissent comme un "umbrella term encompassing all the mimetic transfers that allow different levels of reference to interact and complete each other » (p. 2).

2 Issu du colloque "Choral Mediations in Greek Drama", tenu à la Northwestern University les 30 et 31 Octobre 2009, le volume rassemble quinze études précédées d'un chapitre introductif dans lequel les éditeurs illustrent le concept de «choral mediations " à l'aide du $2^{\mathrm{e}}$ stasimon de l'Électre d'Euripide et retracent les plus 
influentes interprétations du chœur tragique des dernières décennies pour montrer les diverses facettes du questionnement contemporain sur le chœur tragique, de sa dimension socio-politique aux rapports avec la poésie mélique, au domaine rituel. La plasticité et l'étendue possible du concept de «choral mediations » se reflètent dans la grande variété des études rassemblées dans le volume. Il est dès lors impossible d'y trouver une profonde cohérence méthodologique ; le lecteur est appelé plutôt à se faire lui-même «médiateur » entre les différentes pratiques savantes mises en œuvre dans les différents essais.

3 L'essai de Claude Calame qui ouvre le livre offre une réflexion d'ordre théorique et méthodologique. Après avoir discuté plusieurs théories sur le chœur tragique, C.C. reprend et développe l'analyse de la " polyphonie chorale » qu'il a proposée dans des études précédentes ${ }^{2}$, en distinguant entre une voix performative, une voix herméneutique et une voix émotionnelle du chœur. À l'exemple des Perses, il montre la manière dont la polyphonie chorale permet d'articuler la double identité, fictionnelle et rituelle, du chœur. Malgré leur identité dramatique de vieillards perses, les choreutes agissent et jugent en Grecs : si la voix performative et la voix herméneutique sont grecques, c'est à la voix émotionnelle d'articuler le rapport entre Grecs et Perses en faisant de la tragédie une plainte dramatisée qui résout ainsi l'opposition entre les deux identités du chœur.

4 Les deux études suivantes sont également consacrées au théâtre d'Eschyle. Marianne Hopman analyse le chœur des Perses d'un point de vue narratologique. En se fondant sur les catégories d' "Object», "Zooming», «Filter» et "Slant», elle montre la centralité de la voix chorale et son point de vue privilégié par rapport à celui des autres personnages. Elle insiste particulièrement sur la « choral closure » (p. 72) de la tragédie et discute plusieurs exemples dans lesquels le chœur semble unir les autres personnages et le public à ses propres chants à la fin des pièces. Dans «Choral Intemporalities in the Oresteia », Jonas Grethlein étudie les glissements temporels dans la trilogie eschyléenne. Il s'intéresse en particulier aux anachronies présentes dans la parodos de l'Agamemnon, aux gnômai et aux références mythologiques qui permettent de relier des temporalités différentes et de créer des jeux complexes avec l'action mise en scène. Dans la dernière partie de son essai, J.G. analyse le rituel mis en scène à la fin des Euménides et la manière dont il permet d'intégrer les spectateurs dans la performance en superposant les systèmes intérieur et extérieur de communication. "The ritual on the stage, conclut-il, fuses with the ritual of the stage » (p. 98).

5 Dans la seule étude consacrée au théâtre de Sophocle, Simon Goldhill analyse la construction de la voix chorale et l'opposition métrique entre voix lyrique et voix iambique. Il montre en particulier la manière dont Sophocle manipule les passages entre voix parlée et voix chantée, voix individuelle et collective pour accentuer les émotions.

6 Les quatre études suivantes portent sur Euripide. Dans «Conflicting identities in the Euripidean Chorus », Laura Swift s'intéresse à la construction conflictuelle de l'identité des chœurs de Médée et Ion. Dans le premier cas, elle montre de manière convaincante que Médée peut manipuler le chœur de femmes corinthiennes en faisant prévaloir leur identité de genre sur leur identité civique. Dans le deuxième cas, la confusion qu'a le chœur sur sa double identité de membres de la cité d'Athènes et de la famille d'Érechthée montrerait les dangers de l'utilisation de l'identité nationale à des fins particulières. Dans "The choral plot of Euripides' Helen », Sheila Murnaghan analyse 
l'opposition entre personnage et groupe dans l'Hélène en appliquant à sa lecture de la tragédie le paradigme de la jeune fille qui sort du chœur. Pour S.M., le personnage d'Hélène se présente comme un " dislocated chorus leader » (p. 164) qui envisagerait la possibilité de réintégrer le groupe choral avec son retour à Sparte. Dans le chapitre suivant, Barbara Kowalzig livre une stimulante analyse du chœur de l'Iphigénie en Tauride dont elle fait l'exemple de la " transcultural chorality » (p. 179). Dans sa lecture, la pratique chorale n'est pas seulement d'ordre civique et religieux, mais agit comme un médiateur entre les différentes cultures et traditions religieuses. Après avoir rappelé l'importance des chœurs tout autour de la Méditerranée et le rôle joué par la thêoria chorale comme pratique d'échange transculturel, B.K. étudie l'imagerie navale et les nombreuses références aux étrangers dans la pièce euripidéenne. Par la discussion de sources littéraires et de données archéologiques, elle fait ressortir le rôle d'Artémis dans la médiation transculturelle à l'intérieur de la région géographique liée au mythe d'Iphigénie. En reprenant et en approfondissant l'interprétation de JeanPierre Vernant («Artemis has the powers to integrate the wild and civilized within the Greek City », p. 197), B.K. montre que l'étiologie du rituel d'Halai Araphenides est une traduction transculturelle du rituel taurien. De cette manière, toute la tragédie « constitutes a large-scale aetiology for an instance of religious integration between the Athenians and the Taurians» (p. 190) et le chœur, par sa transformation de chœur d'esclaves en chœur de citoyens, et par son voyage de Chersonèse à Halai, joue le rôle $\mathrm{du}$ médiateur transculturel. Une mise en contexte historique rappelle de manière pertinente que la religion a toujours été importante dans les échanges culturels et économiques. Dans le dernier essai consacré à Euripide, Anton Bierl analyse le chœur des Bacchantes du point de vue de la «self-referentiality». Il analyse d'abord la superposition du rôle dramatique et performatif du chœur dans la célébration du dieu, en particulier dans la parodos. À travers l'analyse des projections chorales et des autoréférences, il montre ensuite la manière dont le chœur réalise une médiation entre l'espace scénique et l'espace extra-scénique du Cithéron. Touchant un point controversé de l'interprétation de la pièce, A.B. soutient ainsi que « the female devotees of Dionysos cannot be divided into cultic, positive bacchants and mythic-negative maenads $»\left(\right.$ p. 226) ${ }^{3}$.

7 Les quatre études suivantes sont consacrées à quatre « ancient parallels of tragic choral mediations » (p. 33). Gregory Nagy reprend le célèbre passage que l'Hymne homérique à Apollon consacre aux jeunes filles de Délos et à leur pratique chorale. Il montre que les jeunes filles de Délos entretiennent avec le poète le même rapport qu'ont les Muses de la Théogonie avec Hésiode et constituent le modèle de la mimésis (considérée comme «reenactement ») réalisée par la performance chorale dans la tragédie. L'opposition traditionnelle entre un chœur formé de citoyens et un poète professionnel se retrouve dans la performance de la tragédie qui oppose un chœur citoyen à des acteurs professionnels. Par là le chœur peut réaliser sa fonction médiatrice entre le temps héroïque et le temps du rituel, entre les personnages mythologiques et le public. C'est ainsi que, par la médiation du chœur, le pathos (événement douloureux) du héros devient le pathos (émotion) des spectateurs. L'étude de Laura Prauscello, «Choral persuasion in Plato's Laws", analyse la fonction médiatrice du chœur dans la construction morale et sociale de la cité idéale. La pratique chorale joue en effet un rôle éducatif important car elle permet de transmettre aux citoyens une physiologie des émotions. L'auteur montre ensuite la nécessité de mettre en perspective les positions exprimées dans les Lois avec la pratique théâtrale contemporaine. Deux études sont 
consacrées à la comédie. Dans la première, Jeffrey Henderson analyse les formes comiques de médiation chorale et plus particulièrement la manière dont elle permet de porter la critique politique sur la scène des premières pièces d'Aristophane. Dans la deuxième étude, Renaud Gagné s'intéresse à la Tragédie de l'Alphabet de Callias. Après avoir passé en revue les différents passages tragiques mettant en scène une "performance of writing " (p. 303), R.G. montre la manière dont la pièce de Callias réalisait une interaction unique entre paroles, mouvements et images en mettant en scène le rapport complexe que le théâtre instaure entre vision et audition.

Les trois derniers essais du volume portent sur la réception moderne du chœur. Dans le premier essai, Joshua Bellings s'intéresse aux idéalistes allemands et plus particulièrement à l'influence et au succès de la réflexion de Hölderling et de Hegel sur le chœur comme lieu privilégié de la dialectique tragique. J.B. montre ensuite que leur interprétation du chœur comme expression d'une collectivité reflétait l'intérêt qu'ils portaient aux événements de la Révolution française. L'étude de Fiona Macintosh déplace le lecteur dans l'Angleterre des années 1880-1914. L'auteur reconstruit l'intérêt et la fascination pour les chœurs antiques dans les dernières décennies avant la première guerre mondiale et montre comme cet engouement reflète les préoccupations politiques et morales de l'époque victorienne. L'essai final de Peter Meineck s'intéresse à la manière dont quatre metteurs en scène américains contemporains (Richard Schechner, Mark Adamo, Anne Bogart et Will Power) ont mis en scène le chœur. Entre des entretiens avec les metteurs en scène et de fines réflexions sur les textes, P.M. montre comme la mise en scène du chœur a permis une médiation entre les préoccupations contemporaines et un théâtre qui, par ses formes discursives et par ses conventions scéniques, est profondément éloigné du nôtre. Une riche bibliographie et un index regroupant les principaux noms, lieux et matières closent le livre.

On peut saluer dans l'ensemble la richesse des analyses proposées dans ce volume. Si toutes les études ne semblent pas toujours développer le concept de médiation chorale de manière suffisamment claire et élaborée, l'étendue et la variété des sujets abordés font de ce livre un échantillonnage de possibilités interprétatives et une stimulation à poursuivre la réflexion plutôt que l'exposition cohérente d'une méthode. On aurait cependant aimé trouver dans un chapitre conclusif, ou dans l'introduction des deux éditeurs, une synthèse plus poussée des différentes "médiations " envisagées dans l'ensemble du volume. Une indexation des différents types de médiations chorales discutés aurait également été la bienvenue. Du point de vue formel, le livre présente une remarquable gestion des renvois bibliographiques (avec l'étonnante exception du chapitre 10) et je n'ai remarqué que quelques fautes d'accents (p. $81: \kappa v \varepsilon \varphi \alpha ́$ oṇ pour

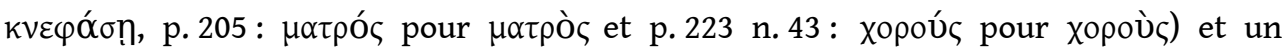

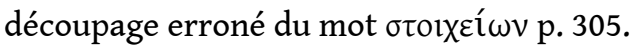

Pour revenir à l'essentiel, le lecteur intéressé à tel ou tel autre aspect de la construction de la voix chorale dans la tragédie grecque pourra trouver dans les essais rassemblés dans ce volume une quantité considérable d'informations et des réflexions riches et stimulantes. La "médiation chorale " y apparaît comme un concept opératoire utilisé de manière peut-être trop malléable, mais les chapitres les plus convaincants montrent qu'il est susceptible d'articuler plusieurs facettes d'une pratique sociale, poétique et religieuse composite comme le chœur et il y a fort à parier que plusieurs études contenues dans ce volume vont devenir des points de repères importants pour les recherches à venir. 


\section{NOTES}

1. C. CALAmE, Les chœurs de jeunes filles en Grèce archaïque, 2 vol., Rome, 1977.

2. Voir notamment C. CALAME, « De la poésie chorale au stasimon tragique. Pragmatique de voix féminines », Métis 12 (1997), p. 181-203.

3. Le point de vue contraire et généralement accepté par la critique est bien présenté par A. HENRICHS, « Die Maenaden von Milet », ZPE 4 (1969), p. 223-241.

\section{AUTEURS}

\section{ROCCO MARSEGLIA}

EPHE - Paris - Centre AnHiMA 